\title{
Kernel-based identification of positive systems
}

\section{Conference Paper}

Author(s):

Khosravi, Mohammad; Smith, Roy (D)

Publication date:

2020

Permanent link:

https://doi.org/10.3929/ethz-b-000388052

Rights / license:

In Copyright - Non-Commercial Use Permitted

Originally published in:

https://doi.org/10.1109/CDC40024.2019.9029276 


\title{
Kernel-Based Identification of Positive Systems
}

\author{
Mohammad Khosravi and Roy S. Smith
}

\begin{abstract}
In this paper, we introduce a novel method for identification of internally positive systems. In this regard, we consider a kernel-based regularization framework. For the existence of a positive realization of a given transfer function, necessary and sufficient conditions are introduced in the realization theory of the positive systems. Utilizing these conditions, we formulate a convex optimization problem by which we can derive a positive system for a given set of inputoutput data. The optimization problem is initially introduced in reproducing kernel Hilbert spaces where stable kernels are used for estimating the impulse response of system. Following that, employing theory of optimization in function spaces as well as the well-known representer theorem, an equivalent convex optimization problem is derived in finite dimensional Euclidean spaces which makes it suitable for numerical simulation and practical implementation. Finally, we have numerically verified the method by means of an example and a Monte Carlo analysis.
\end{abstract}

\section{INTRODUCTION}

The quantities of interest in many systems are constrained to be non-negative, either according to the nature of their definition or the physics of the underlying system, e.g., the pressure of a fluid, the population of certain species of bacteria, the state variables in the compartmental systems like the concentrations of pathogens and the thermal energy in a building, just to name a few [1]-[6]. The systems with quantities of this type are generally called positive systems [6]. In the literature [6], [7], according to the quantities of interest, two main types of positive systems are introduced, externally positive systems and internally positive systems. The externally positive systems are the systems where their output signal has non-negative values for all non-negative input signals. Internally positive systems are the systems in which the non-negativity of the initial state and the input signal leads to non-negativity of the output and the states.

Respecting the positivity property in modeling and identification of positive systems can be an essential issue in many applications such as the implementation of model predictive control [8]. In fact, disregarding this property can produce results or behaviors which are not interpretable or in contradiction with the expected behavior [8]. While the realization theory of positive systems has received significant attention [1], the identification problem of such systems is not well studied. Regarding internally positive systems, conditions for a system being compartmental are presented in [9] for identification. Third order positive systems with a Poisson output are considered in [10]. Also, the stability and scalability of internally positive system identification is

\footnotetext{
*This research project is part of the Swiss Competence Center for Energy Research SCCER FEEB\&D of the Swiss Innovation Agency Innosuisse.

The authors are with Automatic Control Lab, ETH Zurich, Switzerland \{khosravm, rsmith\} dcontrol. ee.ethz.ch
}

discussed in [8], for the specific case where, in addition to the measurement data of input and output of system, the measurement data of state variables is available. The identification of externally positive systems is discussed in [11] and [12]. The method introduced in [11] is based on semidefinite programming and guarantees to identify externally positive models. A kernel-based method is also introduced in [12], where a Bayesian nonparametric approach is utilized for the identification of externally positive systems. The kernel-based methods, beside resolving the bias-variance trade-off issue, provide a framework for considering prior knowledge on the system to be estimated [13]. In these methods, the system identification problem is formulated as a regularized regression problem where a regularization term is considered for penalizing the feasible solutions which do not match the prior knowledge. This prior knowledge can include the stability of the system, the smoothness of the impulse response, time constants and resonant frequencies [14]. According to the realization theory of positive systems [6], a transfer function of an external positive system has an internal positive realization if the transfer function has a special structured form. Given this specific structure, we use a change of variable in order to formulate the identification problem of internal positive systems. Based on the resulting form and using measurement data, we estimate a stable transfer function subject to linear constraints which are imposed by the positivity property of system. This estimation problem is formulated as a regularized optimization problem where stable kernels are utilized.

\section{Notations AND PRELIMINARIES}

\section{A. Notations}

In this paper, the set of natural numbers, the set of integers and the set of real numbers are shown as $\mathbb{N}, \mathbb{Z}$ and $\mathbb{R}$, respectively. For any $1 \leq n \leq \infty$, the vector space of $n$ dimensional vectors with entries in $\mathbb{R}$ is denoted by $\mathbb{R}^{n}$ and $\mathrm{h} \in \mathbb{R}^{n}$ is shown as $\mathrm{h}=\left(h_{k}\right)_{k=1}^{n}$ (or in short form by $\left(h_{k}\right)_{k}$ ) in terms of its entries, i.e., $h_{k}$ is the entry of $\mathrm{h}$ at location $k$. The positive orthant of $\mathbb{R}^{n}$ is denoted by $\mathbb{R}_{>0}^{n}$. Similarly, $\mathbb{R}^{n \times n}$ denotes the space of matrices (operators) from $\mathbb{R}^{n}$ to $\mathbb{R}^{n}$. The identity matrix and zero matrix in $\mathbb{R}^{n \times n}$ are denoted by $\mathbb{I}_{n}$ and $\mathbb{O}_{n}$, respectively. Also, the zero vector and the allone vector in $\mathbb{R}^{n}$ are denoted by $\mathbf{0}_{n}$ and $\mathbf{1}_{n}$, respectively. For any pair of vectors $\mathrm{x}$ and $\mathrm{y}$ in $\mathbb{R}^{n}$, we write $\mathrm{x} \geq \mathrm{y}$ if each entry of $x-y$ is non-negative. Similarly, for any pair of matrices $\mathrm{X}$ and $\mathrm{Y}$ in $\mathbb{R}^{n \times n}$, we write $\mathrm{X} \geq \mathrm{Y}$ if each entry of $\mathrm{X}-\mathrm{Y}$ is non-negative. For any $p \in[1, \infty)$, the $p$-norm of vector $\mathrm{h}=\left(h_{k}\right)_{k} \in \mathbb{R}^{n}$ is defined as $\|\mathrm{h}\|_{p}=\left(\sum_{k}\left|h_{k}\right|^{p}\right)^{\frac{1}{p}}$, and its $\infty$-norm is defined as $\|\mathrm{h}\|_{\infty}=\sup _{k}\left|h_{k}\right|$, where 
$1 \leq n \leq \infty$. The spaces of vectors $\mathrm{h}=\left(h_{k}\right)_{k}$ with finite $p$ norm is denoted by $\ell^{p}$. Given a vector $\mathrm{h}=\left(h_{k}\right)_{k} \in \mathbb{R}^{n}$, we define a Hankel matrix (operator), denoted by Hankel(h), as a matrix (operator) where its entry at location $(i, j)$ is $h_{i+j-1}$. For scalars $w_{1}, \ldots, w_{n}>0$ and $\mathrm{x} \in \mathbb{R}^{n}$, we define a weighted norm, denoted by $\|x\|_{\mathrm{w}}$, as $\left(\sum_{i=1}^{n} w_{i} x_{i}^{2}\right)^{\frac{1}{2}}$ where $\mathrm{x}=\left[x_{1} \ldots x_{n}\right]^{\top}$ and $\mathrm{w}=\left[w_{1} \ldots w_{n}\right]^{\top}$. Given $i \in \mathbb{N}$ and $\mathrm{u}=\left(u_{k}\right)_{k} \in \ell^{\infty}$, we can define a linear operator on $\ell^{1}$, denoted by $\mathrm{L}_{i}^{\mathrm{u}}$, as $\mathrm{L}_{i}^{\mathrm{u}}[\mathrm{h}]=\sum_{k=1}^{i-1} h_{k} u_{i-k}$, for any $\mathrm{h}=\left(h_{k}\right)_{k} \in \ell^{1}$. One can easily see that this is a bounded operator. Let $\mathcal{X}$ be a set and $\mathcal{C}$ be a subset of $\mathcal{X}$. The indicator function of $\mathcal{C}$, denoted by $\mathcal{I}_{\mathcal{C}}$, is defined as $\mathcal{I}_{\mathcal{C}}(x)=0$, if $x \in \mathcal{C}$ and $\mathcal{I}_{\mathcal{C}}(x)=\infty$, otherwise.

\section{B. RKHS, Representer Theorem and System Identification}

In this section, we briefly review main properties of reproducing kernel Hilbert spaces (RKHS) [15]. For a detailed and comprehensive introduction one can see [16].

Let $\mathscr{X}$ be a non-empty set. Denote by $\mathbb{R}^{\mathscr{X}}$ as the vector space of $\mathbb{R}$-valued functions defined over $\mathscr{X}$.

Definition 2.1: A symmetric function $\mathbb{k}: \mathscr{X} \times \mathscr{X} \rightarrow \mathbb{R}$ is a positive definite kernel if we have

$$
\sum_{i=1}^{n} \sum_{j=1}^{n} c_{i} c_{j} \mathbb{k}\left(x_{i}, x_{j}\right) \geq 0,
$$

for any $n \in \mathbb{N}$ and $\left(x_{1}, c_{1}\right), \ldots,\left(x_{n}, c_{n}\right) \in \mathscr{X} \times \mathbb{R}$. For any $x$ in $\mathscr{X}$, the kernel section centered at $x$, denoted by $\mathbb{k}_{x}$, is defined as a function $\mathbb{k}_{x}: \mathscr{X} \rightarrow \mathbb{R}$ where for any $y \in \mathscr{X}$, we have $\mathbb{k}_{x}(y)=\mathbb{k}(x, y)$.

Definition 2.2 (Reproducing Kernel Hilbert Space):

Let $\mathcal{H}_{\mathbb{k}} \subseteq \mathbb{R}^{\mathscr{X}}$ be a Hilbert space with inner product $\langle\cdot, \cdot\rangle_{\mathcal{H}_{\mathbb{k}}}$. We say Hilbert space $\mathcal{H}_{\mathbb{k}}$ is a Reproducing Kernel Hilbert Space, if there exists a positive definite kernel $\mathbb{k}: \mathscr{X} \times \mathscr{X} \rightarrow \mathbb{R}$ such that, for any $(x, f)$ in $\mathscr{X} \times \mathcal{H}_{\mathbb{k}}$, we have

$$
\left\langle\mathbb{k}_{x}, f\right\rangle=f(x) .
$$

The property (2) is called reproducing property.

Theorem 2.3 (Representer Theorem): Let $\mathcal{H}_{k} \subseteq \mathbb{R}^{\mathscr{X}}$ be a RKHS with positive definite kernel $\mathbb{k}: \mathscr{X} \times \mathscr{X} \rightarrow \mathbb{R}$ and $\mathrm{L}_{1}, \ldots, \mathrm{L}_{n}: \mathcal{H}_{\mathbb{k}} \rightarrow \mathbb{R}$ be bounded linear functionals, $f: \mathbb{R}^{n} \rightarrow \mathbb{R} \cup\{+\infty\}$ be a given function and $\gamma>0$ be a positive real scalar. If the optimization problem

$$
\min _{\mathrm{h} \in \mathcal{H}_{\mathbb{k}}} f\left(\mathrm{~L}_{1}[\mathrm{~h}], \mathrm{L}_{2}[\mathrm{~h}], \ldots, \mathrm{L}_{n}[\mathrm{~h}]\right)^{2}+\gamma\|\mathrm{h}\|_{\mathcal{H}_{k}}^{2},
$$

has a solution, then there exists a solution $\mathrm{h}^{*}$ for this problem in the following form

$$
\mathrm{h}^{*}=\sum_{i=1}^{n} c_{i} \mathrm{~L}_{i}[\mathbb{k}] .
$$

Moreover, one has

$$
c \in \operatorname{argmin}_{\mathrm{c} \in \mathbb{R}^{n}} f(\mathrm{Rc})+\gamma \mathrm{c}^{\top} \mathrm{Rc},
$$

where $\mathrm{c}=\left(c_{i}\right)_{i=1}^{n} \in \mathbb{R}^{n}$ and

$$
\mathrm{R}=\left[\left\langle\mathrm{L}_{i}[\mathbb{k}], \mathrm{L}_{j}[\mathbb{k}]\right\rangle_{\mathcal{H}_{k}}\right]_{i, j=1}^{n}=\left[\mathrm{L}_{i}\left[\mathrm{~L}_{j}[\mathbb{k}]\right]\right]_{i, j=1}^{n} \in \mathbb{R}^{n \times n}
$$

Let $\mathscr{X}$ be either the set of natural numbers or $\{1,2, \ldots, n\}$, for a given $n \in \mathbb{N}$. An impulse response of a (strictly) causal system is an element of $\mathbb{R}^{\mathscr{X}}$. By considering a positive-definite kernel $\mathbb{k}: \mathscr{X} \times \mathscr{X} \rightarrow \mathbb{R}$, one obtains a corresponding reproducing kernel Hilbert space, denoted by $\mathcal{H}_{\mathbb{k}}$. Since $\mathcal{H}_{\mathbb{k}}$ is a subset of $\mathbb{R}^{\mathscr{X}}$, each element of $\mathcal{H}_{\mathbb{k}}$ represents an impulse response of a casual discrete time system. However, since we are interested in the identification of stable systems, the kernel $\mathbb{k}$ should produce a RKHS where each of the impulse responses in $\mathcal{H}_{\mathbb{k}}$ is a stable one. In other words, it is desired that for any $\mathrm{f}=\left(f_{k}\right)_{k=0}^{\infty}$ in $\mathcal{H}_{\mathbb{k}}$, one has $\sum_{k=0}^{\infty}\left|f_{k}\right|<\infty$ [13], [17], [18]. The kernels with this property are called stable kernels [13]. Equivalently, we say that $\mathcal{H}_{\mathbb{k}}$ is stable if $\mathcal{H}_{\mathbb{k}} \subseteq \ell^{1}$.

In [13], [17], several stable kernels are introduced including

- Diagonal/Correlated (DC) kernel:

$$
\mathbb{K}^{(\mathrm{DC})}(i, j)=\alpha^{\frac{i+j}{2}} \rho^{|i-j|}, \quad \forall i, j \in \mathscr{X},
$$

where $\alpha \in[0,1)$ and $\rho \in[-1,1]$ are the hyperparameters of $k^{(\mathrm{DC})}$,

- Tuned/Correlated (TC) kernel:

$$
\mathbb{k}^{(\mathrm{TC})}(i, j)=\alpha^{\max (i, j)}, \quad \forall i, j \in \mathscr{X},
$$

where $\alpha \in[0,1)$ is the hyperparameter of $\mathbb{k}^{(\mathrm{TC})}$, and,

- Stable Spline (SS) kernel:

$$
\mathbb{K}^{(\mathrm{SS})}(i, j)=\frac{\alpha^{i+j+\max (i, j)}}{2}-\frac{\alpha^{3 \max (i, j)}}{6}, \quad \forall i, j \in \mathscr{X},
$$

where $\alpha \in[0,1)$ is the hyperparameter of $\mathbb{k}^{(s s)}$.

Definition 2.4: A kernel $\mathbb{k}$ is a finite-rank Hankel kernel if the Hankel operator of each of its sections is a finite rank operator, i.e., for any $k \in \mathscr{X}$, we have

$$
\operatorname{rank}\left(\operatorname{Hankel}\left(\mathbb{k}_{k}\right)\right)<\infty .
$$

One can see that $\mathbb{k}^{(\mathrm{DC})}, \mathbb{k}^{(\mathrm{TC})}$ and $\mathbb{k}^{(\mathrm{SS})}$ are finite-rank Hankel kernels. Moreover, when $\mathscr{X}$ is a finite set, any kernel is a finite-rank Hankel kernel.

\section{The Realization Theory of Positive Systems}

Let $\mathcal{S}$ be a causal discrete-time LTI system with impulse response $\mathrm{g}=\left(g_{k}\right)_{k=0}^{\infty}$, i.e., given that $\mathrm{u}=\left(u_{k}\right)_{k \in \mathbb{Z}}$ is the input of system, the (noiseless) output of system, at time instant $t$, is given by

$$
\mathcal{S}: y_{t}=\sum_{k=0}^{\infty} g_{k} u_{t-k} .
$$

Definition 3.1 ([6]): System $\mathcal{S}$ is an externally positive system if $y_{t} \geq 0$, for all $t$, when we have $u_{t} \geq 0$, for all $t$.

Definition 3.1 says that a system is externally positive if each value of its output is non-negative when the values of its input are all non-negative. The following theorem provides a sufficient and necessary condition for external positivity.

Theorem 3.2 ([6]): System $\mathcal{S}$ is externally positive if and only if $g_{k} \geq 0$, all any $k$. 
According to Theorem 3.2, external positive systems are exactly the systems with non-negative impulse responses. In addition to external positivity, there is another notion of positivity called internal positivity or simply positivity. Let $\mathcal{S}$ has a state-space representation as following:

$$
\mathcal{S}:\left\{\begin{aligned}
\mathrm{x}_{t+1} & =\mathrm{Ax}_{t}+\mathrm{b} u_{t}, \\
\mathrm{y}_{t} & =\mathrm{c}^{\mathrm{T}} \mathrm{x}_{t}+d u_{t},
\end{aligned}\right.
$$

where $\mathrm{A} \in \mathbb{R}^{n_{\mathrm{x}} \times n_{\mathrm{x}}}, \mathrm{b}, \mathrm{c} \in \mathbb{R}^{n_{\mathrm{x}}}$ and $d \in \mathbb{R}$.

Definition 3.3 ([6]): System $\mathcal{S}$ is an internally positive system if $\mathrm{x}_{t} \geq \mathbf{0}$ and $y_{t} \geq 0$, for all $t \geq 0$, when we have $u_{t} \geq 0$, for all $t \geq 0$, and $\mathrm{x}_{0} \geq \mathbf{0}$.

According to Definition 3.3, for internally positive systems, in addition to the output, internal state variables remain non-negative when the input signal is non-negative. The necessary and sufficient condition for internal positivity is given in the following theorem.

Theorem 3.4 ([6]): System $\mathcal{S}$ is (internally) positive if and only if $\mathrm{A} \geq \mathbb{O}_{n_{\mathrm{x}}}, \mathrm{b}, \mathrm{c} \geq \mathbf{0}_{n_{\mathrm{x}}}$ and $d \geq 0$.

Theorem 3.5 ([6]): If system $\mathcal{S}$ is (internally) positive then $g_{k} \geq 0$, for any $k$.

Theorem 3.5 says that any internal positive system is also externally positive, however, the converse is not true. This fact is confirmed by the following example which is a modified version of an example given in [1].

Example 3.6: Let $\alpha$ be a positive real scalar and $g_{k}$ be defined as

$$
g_{k}=\alpha^{k}(1+\cos (2 \pi \omega k))
$$

for any $k \in \mathbb{Z}_{>0}$, where $\omega$ is an irrational number. The impulse response $\mathrm{g}=\left(g_{k}\right)_{k}$ is non-negative and corresponds to a finite-order external positive system, meanwhile there is no finite-order state-space representation satisfying conditions given in Definition 3.3. In other words, g corresponds to an externally positive system with no positive realization.

The following theorem provides a sufficient condition for internally positive realization of an externally positive system. This theorem is used in the next section for identification of positive systems.

Theorem 3.7 ([6]): Let $G(z)$ be a strictly proper rational transfer function of order $n$ with nonnegative impulse response $\mathrm{g}=\left(g_{k}\right)_{k}$. If $G(z)$ has a unique dominant pole $\alpha>0$, then $G(z)$ has a positive realization of some finite order $N \geq n$.

The notions of externally and internally positive continuous time LTI systems are defined similar to Definition 3.1 and Definition 3.3, respectively [6]. Moreover, for continuous time systems, we have arguments similar to the theorems given above. However, the analogous version of Theorem 3.5 provides a necessary and sufficient condition.

Theorem 3.8 ([6]): Let $G(s)$ be a strictly proper rational transfer function of order $n$ with nonnegative continuous time impulse response $\mathrm{g}=\left(g_{t}\right)_{t}$. Then, $G(s)$ has a positive realization of some finite order $N \geq n$ if and only if $G(s)$ has a unique real dominant pole and we have $g_{t}>0$, for any $t>0$.

Remark 3.9: In addition to the conditions given in Theorem 3.7, for the positive realization of discrete time systems other similar conditions are introduced in [6]. Moreover, for the positive realization of continuous-time systems, the necessary and sufficient conditions introduced in Theorem 3.8 are the same as the ones provided in Theorem 3.7. Using analogous arguments, in the following section we focus on the identification of positive systems with the structures given in Theorem 3.7. This case can play the role of a bridge amongst these different cases and provides the potential for further generalization.

In the rest of the paper, we refer to internally positive systems as positive systems.

\section{Positive System IdentificAtion}

Consider a SISO system described by

$$
y_{i}=\sum_{k=1}^{\infty} g_{k} u_{i-k}+n_{i}, \quad \forall i \in \mathbb{N},
$$

where $u_{i}, n_{i}$ and $y_{i}$ are the input, additive noise and measured output of system, respectively, and $g=\left(g_{k}\right)_{k=1}^{\infty}$ is the impulse response of system. For the sake of simplicity, we assume that $u_{i}=0$, for $i \leq 0$. Let $\mathcal{D}_{n}$ be the set of input-output data of system for time instants $1 \leq i \leq n$, i.e., $\mathcal{D}_{n}:=\left\{u_{i}, y_{i} \mid 1 \leq i \leq n\right\}$. Here, the main goal is to estimate $\mathrm{g}=\left(g_{k}\right)_{k=1}^{\infty}$ using $\mathcal{D}_{n}$ such that the resulting transfer function $G(z)=\sum_{k=1}^{\infty} g_{k} z^{-k}$ is guaranteed to have a positive realization and a good fit to the data. One should note that these two features may not be always feasible together and the true system is required to be a positive system in this regard.

Given $\alpha, \rho>0$ and $\mathcal{D}_{n}$, we define set $\mathcal{D}_{n}(\alpha, \rho)$ as $\left\{\bar{u}_{i}, \bar{y}_{i} \mid 1 \leq i \leq n\right\}$ where

$$
\begin{aligned}
& \bar{u}_{i}=\alpha^{-i} u_{i} \\
& \bar{y}_{i}=\rho^{-1} \alpha^{-i} y_{i}-\sum_{k=1}^{\infty} \alpha^{k-i} u_{i-k}
\end{aligned}
$$

Theorem 4.1: Let $\alpha, \rho, \gamma, \bar{w}_{1}, \ldots, \bar{w}_{n}$ be positive scalars and $\mathbb{k}$ be a stable kernel. Consider set $\mathcal{D}_{n}(\alpha, \rho)$ and the following optimization problem

$$
\begin{array}{cl}
\min _{h \in \mathcal{H}_{\mathbb{k}}} & \sum_{i=1}^{n} \bar{w}_{i}\left(\bar{y}_{i}-\mathrm{L}_{i}^{\overline{\mathrm{u}}}[\mathrm{h}]\right)^{2}+\gamma\|\mathrm{h}\|_{\mathcal{H}_{\mathbb{k}}}^{2}, \\
\text { s.t. } & h_{k}+1 \geq 0, \quad \forall k \geq 1, \\
& \operatorname{rank}(\operatorname{Hankel}(\mathrm{h}))<\infty .
\end{array}
$$

If $\mathrm{h}=\left(h_{k}\right)_{k=1}^{\infty}$ is the solution of (16) and $H(z)=$ $\sum_{k=1}^{\infty} h_{k} z^{-k}$, then $H(z)$ is a stable system and $G(z)$ defined as

$$
G(z)=\frac{\rho z^{-1}}{1-\alpha z^{-1}}+\rho H\left(\alpha^{-1} z\right),
$$

has a positive realization.

Proof: Since the rank of the Hankel operator Hankel(h) is finite, $H(z)$ is finite order. Therefore, the orders of $H\left(\alpha^{-1} z\right)$ and $G(z)$ are finite. Moreover, since $\mathbb{k}$ is a stable kernel, $\mathrm{h}$ is stable impulse response and all of the poles of $H(z)$ are inside unit disk. Therefore, the magnitude of poles of $H\left(\alpha^{-1} z\right)$ are strictly smaller than $\alpha$. According to this 
and also (17), one can see that $G(z)$ has a unique dominant pole at $\alpha$. Note that we have

$$
\rho H\left(\alpha^{-1} z\right)=\rho \sum_{k=1}^{\infty} h_{k}\left(\alpha^{-1} z\right)^{-k}=\sum_{k=1}^{\infty} \rho h_{k} \alpha^{k} z^{-k}
$$

If $\mathrm{g}=\left(g_{k}\right)_{k=1}^{\infty}$ is the impulse response of $G(z)$, we have from (17) that

$$
\sum_{k=1}^{\infty} g_{k} z^{-k}=\sum_{k=1}^{\infty} \rho \alpha^{k} z^{-k}+\sum_{k=1}^{\infty} \rho h_{k} \alpha^{k} z^{-k}
$$

Therefore, for any $k$, we have

$$
g_{k}=\rho \alpha^{k}\left(1+h_{k}\right) \text {. }
$$

Subsequently, $g_{k} \geq 0$, for any $k$. This shows that $G(z)$ has a unique dominant pole $\alpha>0$ and a nonnegative impulse response. Therefore, according to Theorem 3.7, $G(z)$ has a positive realization.

Remark 4.2: One can easily see that if $w_{i}=\alpha^{-2 i} \bar{w}_{i}$, then, according to (15), we have

$$
\sum_{i=1}^{n} w_{i}\left(y_{i}-\mathrm{L}_{i}^{\mathrm{u}}[\mathrm{g}]\right)^{2}=\sum_{i=1}^{n} \bar{w}_{i}\left(\bar{y}_{i}-\mathrm{L}_{i}^{\overline{\mathrm{u}}}[\mathrm{h}]\right)^{2}
$$

where $\mathrm{g}=\left(g_{k}\right)_{k=1}^{\infty}$ is an impulse response such that $g_{k}=\rho \alpha^{k}+\rho h_{k}$, for all $k \geq 1$. In other words, the first term in cost function of optimization in (16) corresponds to a weighted sum-of-squares error for fitting the impulse response of system given that the dominant pole is known.

Note that from Theorem 3.7, one can see that if transfer function $G(z)$ satisfies (17) and the corresponding impulse response is non-negative, then $G(z)$ has a positive realization. Conversely, if a system is positive and it has a unique dominant pole $\alpha$, then its transfer function $G(z)$ satisfies (17) for some $\rho>0$ and a stable transfer function $H(z)$ with impulse response $\mathrm{h}=\left(h_{k}\right)_{k=1}^{\infty}$ such that $h_{k}+1 \geq 0$, for any $k$. Accordingly, for identification of a positive system with a unique dominant pole, it is enough to obtain a stable impulse response such that $h_{k}+1 \geq 0$, for any $k$, given that $\alpha$, the dominant pole of system, and $\rho$ are known in (17). This is in fact the goal of the optimization problem (16) introduced in Theorem 4.1. This optimization problem is in an infinite-dimensional space with infinite number of constraints. In the following, we analyze this problem and provide a tractable approach for deriving its solution.

Remark 4.3: Here, one can consider finite impulse responses just by assuming that the kernel is defined on the finite set $\mathcal{X}=\{1,2, \ldots, N\}$. However, for the sake of generality and also the potential of generalizing the theory to the case of continuous time positive systems, we set $\mathcal{X}$ as $\mathcal{X}=\mathbb{N}$.

Define the set $\mathcal{F} \subseteq \mathcal{H}_{\mathbb{k}}$ as $\left\{\mathrm{h} \in \mathcal{H}_{\mathbb{k}} \mid \operatorname{rank}(\operatorname{Hankel}(\mathrm{h}))<\right.$ $\infty\}$ and set $\mathcal{P}_{k} \subseteq \mathcal{H}_{\mathbb{k}}$ as $\mathcal{P}_{k}:=\left\{\mathrm{h} \in \mathcal{H}_{\mathbb{k}} \mid h_{k}+1 \geq 0\right\}$. Now, let $J_{\mathcal{F}}: \mathcal{H}_{\mathbb{k}} \rightarrow \mathbb{R} \cup\{+\infty\}$ be defined as

$$
J_{\mathcal{F}}(\mathrm{h})=\sum_{i=1}^{n} \bar{w}_{i}\left(\bar{y}_{i}-\mathrm{L}_{i}^{\overline{\mathrm{u}}}[\mathrm{h}]\right)^{2}+\sum_{k=1}^{\infty} \mathcal{I}_{\mathcal{P}_{k}}(\mathrm{~h})+\mathcal{I}_{\mathcal{F}}(\mathrm{h})+\gamma\|\mathrm{h}\|_{\mathcal{H}_{\mathbb{k}}}^{2} .
$$

Then, one can easily see that optimization problem (16) is equivalent to

$$
\inf _{\mathrm{h} \in \mathcal{H}_{\mathbb{k}}} J_{\mathcal{F}}(\mathrm{h}) .
$$

Since, for $\mathrm{h}=\mathbf{0}$ we have

$$
J_{\mathcal{F}}(\mathbf{0})=\sum_{i=1}^{n} \bar{w}_{i} \bar{y}_{i}^{2}<\infty
$$

we know that $\inf _{\mathrm{h} \in \mathcal{H}_{k}} J_{\mathcal{F}}(\mathrm{h})<\infty$. Now, define function $J: \mathcal{H}_{\mathbb{k}} \rightarrow \mathbb{R} \cup\{+\infty\}$ as

$$
J(\mathrm{~h})=\sum_{i=1}^{n} \bar{w}_{i}\left(\bar{y}_{i}-\mathrm{L}_{i}^{\overline{\mathrm{u}}}[\mathrm{h}]\right)^{2}+\sum_{k=1}^{\infty} \mathcal{I}_{\mathcal{P}_{k}}(\mathrm{~h})+\gamma\|\mathrm{h}\|_{\mathcal{H}_{\mathbb{k}}}^{2} .
$$

Since $J_{\mathcal{F}}=J+\mathcal{I}_{\mathcal{F}}$, we have that $J(\mathrm{~h}) \leq J_{\mathcal{F}}(\mathrm{h})$, for all $\mathrm{h} \in \mathcal{H}_{\mathbb{k}}$. Therefore, if $\mathrm{h}^{*}$ is a solution of

$$
\min _{\mathrm{h} \in \mathcal{H}_{\mathbb{k}}} J(\mathrm{~h}),
$$

such that rank of Hankel operator Hankel( $\left.h^{*}\right)$ is finite, then it is a solution of (23), and subsequently a solution of (16). In the following, we show that such solution exists if the kernel $\mathbb{k}$ satisfies certain properties. Towards this, we present the following theorem on the existence and uniqueness of the solution of (26).

Theorem 4.4: The optimization problem (26) has a unique solution, i.e., there exists a unique $h^{*}$ in $\mathcal{H}_{\mathbb{k}}$ where for any $\mathrm{h}$ in $\mathcal{H}_{\mathbb{k}}$ such that $\mathrm{h} \neq \mathrm{h}^{*}$, we have

$$
J\left(\mathrm{~h}^{*}\right)<J(\mathrm{~h}) \text {. }
$$

The proof is omitted due to space limitations.

Though optimization problem (26) admits a unique solution, it is in infinite dimensional space and deriving its solution is not straightforward. Meanwhile, since $\mathrm{h}^{*}$ is in $\mathcal{H}_{\mathbb{k}}$ where $\mathbb{k}$ is a stable kernel, it is expected that for large enough $m$, one has $\mathcal{I}_{\mathcal{P}_{m}}\left(\mathrm{~h}^{*}\right)=0$. Toward formalizing this idea, we define function $J_{m}: \mathcal{H}_{\mathbb{k}} \rightarrow \mathbb{R} \cup\{+\infty\}$ as

$$
J_{m}(\mathrm{~h})=\sum_{i=1}^{n} \bar{w}_{i}\left(\bar{y}_{i}-\mathrm{L}_{i}^{\overline{\mathrm{u}}}[\mathrm{h}]\right)^{2}+\sum_{k=1}^{m} \mathcal{I}_{\mathcal{P}_{k}}(\mathrm{~h})+\gamma\|\mathrm{h}\|_{\mathcal{H}_{\mathfrak{k}}}^{2},
$$

and we consider following optimization problem

$$
\min _{\mathrm{h} \in \mathcal{H}_{k}} J_{m}(\mathrm{~h}) .
$$

Note that (29) is equivalent to

$$
\begin{array}{cl}
\min _{h \in \mathcal{H}_{k}} & \sum_{i=1}^{n} \bar{w}_{i}\left(\bar{y}_{i}-\mathrm{L}_{i}^{\overline{\mathrm{u}}}[\mathrm{h}]\right)^{2}+\gamma\|\mathrm{h}\|_{\mathcal{H}_{k}}^{2}, \\
\text { s.t. } & h_{k}+1 \geq 0, \quad k=1, \ldots, m .
\end{array}
$$

Regarding this optimization problem, one may ask about the existence and uniqueness of the solution. These are shown in the following theorem.

Theorem 4.5: For any $m \in \mathbb{N}$, optimization problem (29) has a unique solution $\mathrm{h}^{(m)}$ in $\mathcal{H}_{\mathbb{k}}$.

The proof is omitted due to space limitations.

Once we have a guarantee of the existence of solutions for (29) a natural question arises about the asymptotic behavior of $\mathrm{h}^{(m)}$ and its connection to $\mathrm{h}^{*}$. The following theorem 
clarifies the relation between $\mathrm{h}^{*}$ and $\mathrm{h}^{(m)}$, specifically, for the case where $m$ goes to infinity.

Theorem 4.6: For the unique minimizers, $\mathrm{h}^{(m)}$ of (29) and $\mathrm{h}^{*}$ of (26), the following holds:

i) There exist an integer $m$ such that $\mathrm{h}^{(m)}=\mathrm{h}^{*}$.

ii) For any integer $m$, we have that $\mathrm{h}^{(m)}=\mathrm{h}^{*}$ if and only if $J\left(\mathrm{~h}^{(m)}\right)<\infty$.

iii) If for some integer $m$ we have that $\mathrm{h}^{(m)}=\mathrm{h}^{*}$, then for any $\bar{m} \geq m$, one has $\mathrm{h}^{(\overline{\mathrm{m}})}=\mathrm{h}^{*}$.

The proof is omitted due to space limitations.

The following corollary is based on Theorem 4.6.

Corollary 4.7: There exists a critical integer $m^{*}$ such that for any $m \geq m^{*}$, we have $\mathrm{h}^{(m)}=\mathrm{h}^{*}, J\left(\mathrm{~h}^{(m)}\right)<\infty$ and $h_{k}^{(m)}+1 \geq 0$, for any $k$, where $\mathrm{h}^{(m)}=\left(h_{k}^{(m)}\right)_{k}$. Moreover, for any $m<m^{*}$, we have $\mathrm{h}^{(m)} \neq \mathrm{h}^{*}, J\left(\mathrm{~h}^{(m)}\right)=\infty$ and $h_{k}^{(m)}+1<0$, for some $k$, where here $\mathrm{h}^{(m)}=\left(h_{k}^{(m)}\right)_{k}$.

Next theorem is a representer theorem for problem (29). This is a critical step toward a practical approach for solving optimization problem (16).

Theorem 4.8: For any positive integer $m$, there exist $c_{1}, \ldots, c_{n}, d_{1}, \ldots, d_{m}$ such that $\mathrm{h}^{(m)}=\left(h_{k}^{(m)}\right)_{k}$, the unique solution of (29), admits the following representation

$$
h_{k}^{(m)}=\sum_{i=1}^{n} c_{i} \mathrm{~L}_{i}^{\overline{\mathrm{u}}}\left[\mathbb{k}_{k}\right]+\sum_{j=1}^{m} d_{j} \mathbb{k}_{j}(k) .
$$

Moreover, we have

$$
\begin{array}{ll}
(\mathrm{c}, \mathrm{d})= & \underset{\mathrm{c} \in \mathbb{R}^{n}, \mathrm{~d} \in \mathbb{R}^{m}}{\operatorname{argmin}} \\
\text { subject to } & {\left[\begin{array}{ll}
\mathrm{L}^{\top} & \mathrm{K}
\end{array}\right]\left[\begin{array}{l}
\mathrm{c} \\
\mathrm{d}
\end{array}\right]+\mathbf{1} \geq 0,}
\end{array}
$$

where $\mathrm{c} \in \mathbb{R}^{n}, \mathrm{~d} \in \mathbb{R}^{m}, \overline{\mathrm{w}} \in \mathbb{R}^{n}$ and $\overline{\mathrm{y}} \in \mathbb{R}^{n}$ are vectors defined as

$$
\begin{aligned}
\mathrm{c} & =\left[\begin{array}{llll}
c_{1} & c_{2} & \ldots & c_{n}
\end{array}\right]^{\top}, \\
\mathrm{d} & =\left[\begin{array}{llll}
d_{1} & d_{2} & \ldots & d_{m}
\end{array}\right]^{\top}, \\
\overline{\mathrm{y}} & =\left[\begin{array}{llll}
\bar{y}_{1} & \bar{y}_{2} & \ldots & \bar{y}_{n}
\end{array}\right]^{\top}, \\
\overline{\mathrm{w}} & =\left[\begin{array}{llll}
\bar{w}_{1} & \bar{w}_{2} & \ldots & \bar{w}_{n}
\end{array}\right]^{\top},
\end{aligned}
$$

and matrices $\mathrm{O} \in \mathbb{R}^{n \times n}, \mathrm{~L} \in \mathbb{R}^{n \times m}$ and $\mathrm{K} \in \mathbb{R}^{m \times m}$ are defined as

$$
\begin{array}{lll}
\mathrm{O}\left(i, i^{\prime}\right) & =\mathrm{L}_{i}^{\overline{\mathrm{u}}}\left[\mathrm{L}_{i^{\prime}}^{\overline{\mathrm{u}}}[\mathbb{k}]\right], & 1 \leq i, i^{\prime} \leq n, \\
\mathrm{~L}(i, j) & =\mathrm{L}_{i}^{\mathrm{u}}\left[\mathbb{k}_{j}^{\prime}\right], & 1 \leq i \leq n, 1 \leq j \leq m, \\
\mathrm{~K}\left(j, j^{\prime}\right)=\mathbb{k}\left(j, j^{\prime}\right), & 1 \leq j, j^{\prime} \leq m .
\end{array}
$$

The proof is omitted due to space limitations.

Theorem 4.8 provides a practical way of deriving the solution of (29). According to Theorem 4.6 and Corollary 4.7, this solution is also the solution of (26) if and only if $m \geq m^{*}$. However, optimization problem (26) differs with (23) in one constraint which is the finiteness of the rank of resulting Hankel operator.
Theorem 4.9: If $\mathbb{k}$ is a finite-rank Hankel kernel, then the rank of Hankel $\left(\mathrm{h}^{(m)}\right)$ is finite. Moreover, if $m \geq m^{*}$, we have that $h^{(m)}$, the solution of (29), is also a solution of (16). The proof is omitted due to space limitations.

\section{Numerical VERIFICATIONS AND RESUlts}

In this section, we provide the results of numerical simulations of the discussed method. Here, we have a vector of hyperparameters, denoted by $\theta$, which is $\theta=[\alpha, \rho, \eta]$ where $\eta$ contains the hyperparameters corresponding to the kernel. In order to estimate $\theta$, one can incorporate a crossvalidation $(\mathrm{CV})$ procedure. More precisely, we split the data into two sets; estimation and validation. Then, for a given vector $\theta$, one can estimate the impulse response and predict the values of output for time instants corresponding to the validation set. Let the cross-validation error, denoted by $\operatorname{err}_{\mathrm{CV}}(\theta)$, be defined as the norm of the prediction error vector. In other word, $\operatorname{err}_{\mathrm{CV}}(\theta)$ is the norm of the vector of differences between the true values and the predict ones. Now, one can estimate $\theta$ by minimizing $\operatorname{err}_{\mathrm{CV}}(\theta)$, i.e., $\hat{\theta}=\operatorname{argmin}_{\theta} \operatorname{err}_{\mathrm{CV}}(\theta)$. This optimization problem can be effectively solved by meta-heuristic methods such as genetic algorithm or particle swarm [19]. In each of the numerical examples discussed here, we have used this cross-validation approach for estimating the vector of hyperparameters.

We first discuss a modified version of Example 3.6 and then present the numerical analysis based on the Monte-Carlo procedure. For both cases, we use the TC kernel. Moreover, the results are compared with standard and regularized least squares.

\section{A. Modified Version of Example 3.6}

Example 3.6, introduced in Section III, does not have a positive realization. Here, we consider a modified version of this example. In this regard, let $\alpha, \beta \in(0,1)$ and $g_{k}$ be defined as

$$
g_{k}=\alpha^{k}\left(1+\beta^{k} \cos (2 \pi \omega k)\right),
$$

for any $k \in \mathbb{N}$. One can easily see that for any $k$, we have $g_{k} \geq 0$ and the resulting transfer function is

$$
G(z)=\frac{z^{-1}}{1-\alpha z^{-1}}+\frac{z^{-1}\left(1-\alpha \beta \cos w z^{-1}\right)}{1-2 \alpha \beta \cos w z^{-1}+\alpha^{2} \beta^{2} z^{-2}} .
$$

From Theorem 3.7, one can easily see that $G(z)$ has a positive realization. The structure of this example follows the same line of argument given in the previous section. For the purpose of numerical simulation, we set $\alpha=\beta=0.9$ and $2 \pi w=0.25$. The input $\mathrm{u}$ is chosen as a realization sequence $u_{1}, \ldots, u_{100}$ of independent and identically distributed random Gaussian variables with distribution $\mathcal{N}(0,1)$. Moreover, we consider an additive Gaussian white output noise which is also a realization sequence of independent and identically distributed random Gaussian variables with distribution $\mathcal{N}(0,0.08)$. The noiseless and noisy outputs are shown in Figure 1. Using the input-output data and based on the introduced approach, we estimate the impulse response of this system. As shown in Figure 1, the new method outperforms 

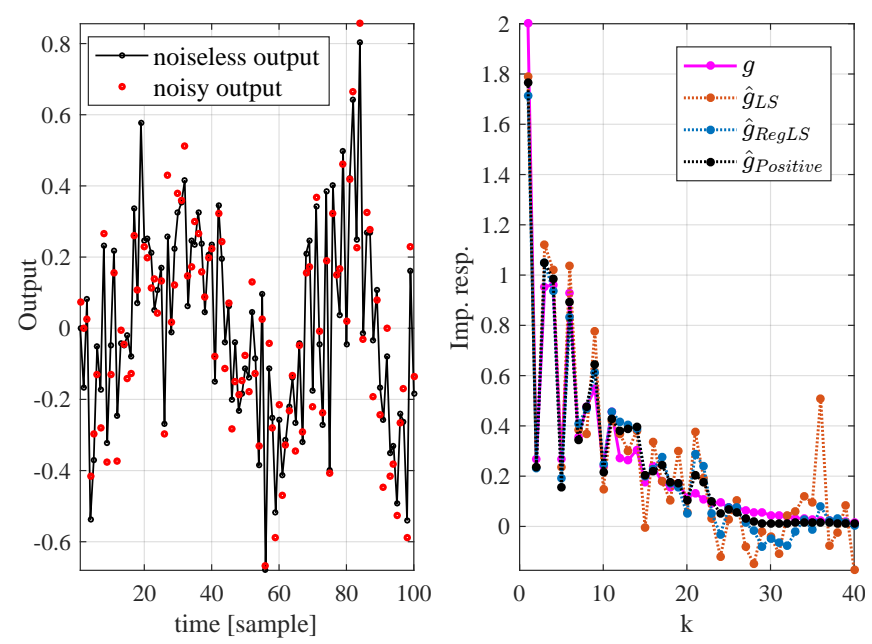

Fig. 1. Output data (left) and different estimations of the impulse response (right).

standard and regularized least square methods. Moreover, one can see that the other approaches fail to provide positive systems.

\section{B. Monte Carlo Simulation}

For Monte Carlo analysis, we randomly generate 300 causal stable positive systems. The orders of systems are uniformly chosen from the set $\{20,21, \ldots, 30\}$. To have fair comparison the systems are normalized by their system norm. For both the input and the output noise, we take a realization sequence of independent and identically distributed zero-mean Gaussian random variables. For the fitness metric, we consider the first $N$ terms of the estimated impulse response, $\hat{g}$, and define

$$
\operatorname{Fitness}(\hat{\mathrm{g}})=1-\frac{\|\hat{\mathrm{g}}-\mathrm{g}\|_{1}}{N\|\mathrm{~g}\|_{1}}
$$

where $\mathrm{g}$ is the true impulse response. In our simulations, we set $N=70$. As it is shown in Figure 2, the positive identification method outperforms the other approaches. Meanwhile, least square violates the positivity property in $100 \%$ of the cases and regularized least square violates this property in approximately $73.7 \%$ of the cases.

\section{CONCLUSION}

We have developed a novel method for the identification of discrete time positive systems using their realization theory and an appropriate change of variable. Accordingly, we have formulated a convex optimization via kernel-based regularization framework using stable and finite-rank Hankel kernels. The existence as well as the uniqueness of solution for this optimization problem is theoretically proved. Moreover, we have developed a practical approach for deriving this solution using a representer theorem in reproducing kernel Hilbert spaces. The numerical simulations verify the performance of our method.

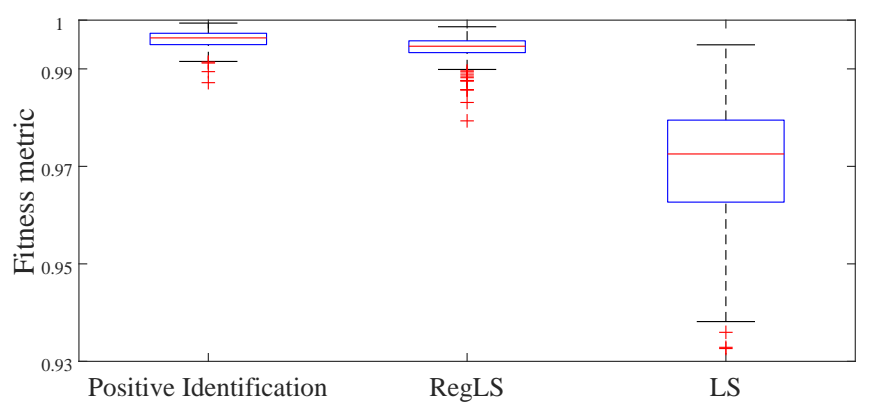

Fig. 2. Monte Carlo results

\section{REFERENCES}

[1] L. Benvenuti and L. Farina, "A tutorial on the positive realization problem," IEEE Transactions on Automatic Control, vol. 49, no. 5, pp. 651-664, 2004.

[2] R. F. Brown, "Compartmental system analysis: State of the art," IEEE Transactions on Biomedical Engineering, no. 1, pp. 1-11, 1980.

[3] R. Shorten, F. Wirth, and D. Leith, "A positive systems model of tcplike congestion control: asymptotic results," IEEE/ACM Transactions on Networking, vol. 14, no. 3, pp. 616-629, 2006.

[4] J. F. Villena and L. M. Silveira, "Circuit synthesis for guaranteed positive sparse realization of passive state-space models," IEEE Transactions on Circuits and Systems I: Regular Papers, vol. 64, no. 6, pp. 1576-1587, 2017.

[5] A. Rantzer and M. E. Valcher, "A tutorial on positive systems and large scale control," in IEEE Conference on Decision and Control (CDC), 2018, pp. 3686-3697.

[6] L. Farina and S. Rinaldi, Positive linear systems: theory and applications. John Wiley \& Sons, 2011, vol. 50.

[7] B. Shafai, J. Chen, and M. Kothandaraman, "Explicit formulas for stability radii of nonnegative and Metzlerian matrices," IEEE Transactions on Automatic Control, vol. 42, no. 2, pp. 265-270, 1997.

[8] J. Umenberger and I. R. Manchester, "Scalable identification of stable positive systems," in IEEE 55th Conference on Decision and Control (CDC), 2016, pp. 4630-4635.

[9] L. Benvenuti, A. De Santis, and L. Farina, "On model consistency in compartmental systems identification," Automatica, vol. 38, no. 11, pp. 1969-1976, 2002.

[10] A. De Santis and L. Farina, "Identification of positive linear systems with poisson output transformation," Automatica, vol. 38, no. 5, pp. 861-868, 2002.

[11] C. Grussler, J. Umenberger, and I. R. Manchester, "Identification of externally positive systems," in IEEE 56th annual conference on decision and control (CDC), 2017, pp. 6549-6554.

[12] M. Zheng and Y. Ohta, "Positive FIR system identification using maximum entropy prior," IFAC-PapersOnLine, vol. 51, no. 15, pp. 7-12, 2018.

[13] G. Pillonetto, F. Dinuzzo, T. Chen, G. De Nicolao, and L. Ljung, "Kernel methods in system identification, machine learning and function estimation: A survey," Automatica, vol. 50, no. 3, pp. 657-682, 2014.

[14] T. Chen, "On kernel design for regularized LTI system identification," Automatica, vol. 90, pp. 109-122, 2018.

[15] N. Aronszajn, "Theory of reproducing kernels," Transactions of the American Mathematical Society, vol. 68, no. 3, pp. 337-404, 1950.

[16] A. Berlinet and C. Thomas-Agnan, Reproducing kernel Hilbert spaces in probability and statistics. Springer Science \& Business Media, 2011.

[17] F. Dinuzzo, "Kernels for linear time invariant system identification," SIAM Journal on Control and Optimization, vol. 53, no. 5, pp. 32993317, 2015.

[18] T. Chen and G. Pillonetto, "On the stability of reproducing kernel Hilbert spaces of discrete-time impulse responses," Automatica, vol. 95, pp. 529-533, 2018.

[19] X.-S. Yang, Engineering optimization: an introduction with metaheuristic applications. John Wiley \& Sons, 2010. 